\title{
Adiponectin inhibits oxidative/nitrative stress during myocardial ischemia and reperfusion via PKA signaling.
}

\author{
Yanqing Zhang \\ Shanxi Medical University \\ Xiao-Liang Wang \\ Shanxi Medical University \\ Jianli Zhao \\ Shanxi Medical University \\ Ya-Jing Wang \\ Shanxi Medical University; Thomas Jefferson University

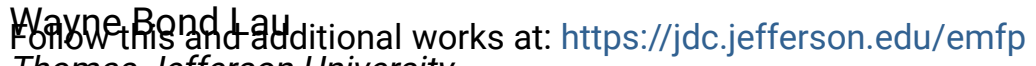 \\ Thomas Jefferson University \\ Part of the Emergency Medicine Commons

\section{Let us know how access to this document benefits you}

\section{Recommended Citation}

Zhang, Yanqing; Wang, Xiao-Liang; Zhao, Jianli; Wang, Ya-Jing; Lau, Wayne Bond; Yuan, Yue-Xing; Gao, Er-He; Koch, Walter J.; and Ma, Xin-Liang, "Adiponectin inhibits oxidative/nitrative stress during myocardial ischemia and reperfusion via PKA signaling." (2013). Department of Emergency Medicine Faculty Papers. Paper 152.

https://jdc.jefferson.edu/emfp/152

This Article is brought to you for free and open access by the Jefferson Digital Commons. The Jefferson Digital Commons is a service of Thomas Jefferson University's Center for Teaching and Learning (CTL). The Commons is a showcase for Jefferson books and journals, peer-reviewed scholarly publications, unique historical collections from the University archives, and teaching tools. The Jefferson Digital Commons allows researchers and interested readers anywhere in the world to learn about and keep up to date with Jefferson scholarship. This article has been accepted for inclusion in Department of Emergency Medicine Faculty Papers by an authorized administrator of the Jefferson Digital Commons. For more information, please contact: JeffersonDigitalCommons@jefferson.edu. 


\section{Authors}

Yanqing Zhang, Xiao-Liang Wang, Jianli Zhao, Ya-Jing Wang, Wayne Bond Lau, Yue-Xing Yuan, Er-He Gao, Walter J. Koch, and Xin-Liang Ma 
Adiponectin Inhibits Oxidative/Nitrative Stress during Myocardial Ischemia and Reperfusion via PKA Signaling

Running Head: PKA in APN's cardioprotective signaling

Yanqing Zhang, MD ${ }^{1}$, Xiao-Liang Wang, M.D., Ph.D..$^{2 *}$, Jianli Zhao, MD ${ }^{1}$, Ya-Jing Wang,M.D.,Ph.D. ${ }^{2,3}$, Wayne Bond Lau,M.D. ${ }^{3}$, Yue-Xing Yuan,Ph.D. ${ }^{3}$, Er-He Gao, M.D., Ph.D. ${ }^{4}$, Walter J Koch, Ph.D. ${ }^{4}$, Xin-Liang Ma, M.D., Ph.D. ${ }^{3 *}$

${ }^{1}$ Department of Anesthesiology, ${ }^{2}$ Department of Physiology, Key Laboratory of Cellular Physiology, Ministry of Education, Shanxi Medical University, Taiyuan, P.R. China 030001, ${ }^{3}$ Department of Emergency Medicine, Thomas Jefferson University, Philadelphia, PA 19107

${ }^{4}$ Center for Translational Medicine, Temple University, Philadelphia, PA 19122

17

${ }^{*}$ Corresponding Authors:

Xin-Liang Ma, M.D., Ph.D

Department of Emergency Medicine, 1025 Walnut Street

College Bldg., Suite 808,

Thomas Jefferson University,

Philadelphia, PA 19107

Tel: 215-955-4994

Fax: 215-923-6225

E-mail: Xin.Ma@Jefferson.edu

Or

Xiaoliang Wang, MD, PhD

Department of Physiology,

Key Laboratory of Cellular Physiology, Ministry of Education,

Shanxi Medical University,

Taiyuan, P.R. China 030001.

E-mail: tywangxl@gmail.com 
Abstract

The cardioprotective effects of adiponectin (APN) against myocardial ischemia/reperfusion $(\mathrm{MI} / \mathrm{R})$ injury are well known. However, comprehension of the mechanisms mediating

41 intracellular APN signaling remains incomplete. We recently demonstrate the antioxidant/antinitrative effects of APN are not dependent upon AMPK. Protein Kinase A (PKA) has been previously shown to be activated by APN, with uncertain relevance to APN reperfusion significantly enhanced cardiac PKA activity, reduced oxidative stress and decreased infarct size. Knockdown of cardiac PKA expression (PKA-KD) by intramyocardial injection of PKA-siRNAs (>70\% suppression) significantly inhibited APN cardioprotection determined by cardiac apoptosis, infarct size, and cardiac function. Moreover, PKA-KD 50 virtually abolished the suppressive effect of APN on MI/R induced NADPH oxidase nitrative protein modification in MI/R heart. Mechanistically, APN significantly inhibited MI/R-induced IKK/I $\mathrm{B}$ phosphorylation and $\mathrm{NF \kappa B}$ activation, which were blocked in PKA-KD heart. Finally, the PKA-mediated antioxidant/antinitrative and cardioprotective effects of APN are intact in AMPK deficiency mice, suggesting that there is no cross-talk between AMPK and PKA signaling in APN cardioprotection. Collectively, we demonstrate

57 for the first time that APN inhibits oxidative/nitrative stress during MI/R via PKA-dependent NFאB inhibition.

60 Keywords: Protein Kinase A; Adiponectin; Reperfusion Injury; Oxidative Stress; 
61 The adipokine adiponectin (APN), of primarily adipose origin, regulates glucose and lipid metabolism(23). Prospective clinical observations and animal studies have demonstrated that reduced adiponectin function may be a major contributor to increased ischemic heart disease morbidity in diabetic patients(21). Administration of the adiponectin attenuates myocardial ischemia and reperfusion (MI/R) injury in mice(20; 24). However, the detailed molecular mechanisms responsible for the cardioprotective effect of adiponectin remain elusive. role in MI/R injury. Superoxide reacts with nitric oxide (NO) to form the highly cytotoxic molecule peroxynitrite $\left(\mathrm{ONOO}^{-}\right)$, which induces nitrative stress and exacerbates $\mathrm{MI} / \mathrm{R}$ injury.

We demonstrated that APN deficient mice exhibited much more severe I/R injury from overproduction of superoxide, peroxynitrite, and resultant nitrative stress, reversible by gAPN administration (22). Therefore, APN is cardioprotective against ischemia/reperfusion injury by inhibiting oxidative/nitrative stress. We have recently demonstrated that the anti-oxidative/anti-nitrative property of APN is not mediated by AMP activated protein kinase (AMPK), the most recognized APN intracellular signaling molecule (24). A comprehensive understanding of intracellular APN anti-oxidative signaling mechanisms remains lacking.

Protein kinase A (PKA) is an important mediator of signal transduction downstream of G-protein-coupled receptors, and plays a key role in the regulation of metabolism and triglyceride storage. In endothelial cells, APN inhibits palmitate-induced apoptosis by suppressing reactive oxygen species (ROS) generation via PKA pathways (27). However, whether PKA signaling mediates APN's anti-oxidative/anti-nitrative effect during MI/R injury has never been previously investigated.

Therefore, the aims of the present study were to investigate whether PKA mediates APN's anti-oxidative/anti-nitrative and cardioprotective effects against MI/R injury; and if so 
to identify the possible responsible intracellular signaling mechanisms.

87 


\section{Materials and Methods}

Adult male WT mice, adult male AMPK-DN mice or their male littermate controls were utilized in this study. Generation, breeding, phenotype characteristics, and genotyping of AMPK-DN mice ( $>80 \%$ inhibition of cardiac AMPK activity) have been previously described in detail (28). The experiments were performed in adherence with the National Institutes of Health Guidelines on the Use of Laboratory Animals and were approved by the Thomas Jefferson University Committee on Animal Care.

Myocardial ischemia/reperfusion: Mice were anesthetized with $2 \%$ isoflurane. MI/R was induced by temporarily exteriorizing the heart via a left thoracic incision, and placing a 6-0 silk suture slipknot around the left anterior descending coronary artery. Twenty minutes after MI, animals were randomized to receive either vehicle or gAPN (2 $\mu \mathrm{g} / \mathrm{g}$, ip) (24). After 30 minutes of MI, the slipknot was released, and the myocardium was reperfused for 3 hours (all assays except cardiac function and infarct size) or 24 hours (cardiac function and infarct size only). All assays were performed utilizing tissue from the ischemic/reperfused area, the area at risk (AAR), identified by Evans blue negative staining. Sham-operated control mice (Sham MI/R) underwent the same surgical procedure, except the suture placed under the left coronary artery was not tied.

Inhibition of PKA with in vivo siRNAs-mediated knock-down (PKA-KD): To specifically confirm the role of PKA in gAPN' protective effect on MI/R, siRNA gene silencing technique was utilized to knock-down mouse cardiac PKA expression (PKA-KD). In brief, two mouse-specific PKA $\alpha$ subunit-selective siRNA targeting the nucleotide sequences 5'-CGTCCTGACCTTTGAGTATCT-3' and 5'-CAGTGTGCTGTTGTAAACATA-3' were employed. siRNA oligos of the same size possessing scrambled nucleotide sequences served as control. All siRNAs were obtained from Integrated DNA Technologies. The siRNAs were diluted in 5\% glucose and mixed with in vivo-jet PEI (Genesee Scientific). Adult mice were 
113 anesthetized with $2 \%$ isoflurane. The heart was exposed via left thoracotomy at the fifth 114 intercostal space. $20 \mu \mathrm{l}$ PKA-specific siRNAs (dose $0.8 \mu \mathrm{g} / \mu \mathrm{l}$ ) or scrambled control was 115 delivered via three separate intramyocardial injections (by $32 \mathrm{G}$ needle), temporarily 116 blanching the left ventricular free wall. 48 hours after siRNAs injection, the mice were 117 subjected to $\mathrm{MI} / \mathrm{R}$.

118 Determination of myocardial apoptosis and myocardial infarct size: Myocardial apoptosis 119 was determined by terminal deoxynucleotidyl transferase-mediated dUTP nick-end labeling 120 (TUNEL) staining and caspase-3 activity as described in our previous study (24). Myocardial 121 infarct size was assessed by Evans blue-2,3,5-triphenyl tetrazolium chloride double staining 122 methods (24).

123 Determination of cardiac function: Cardiac function was determined by echocardiography 124 and left ventricular catheterization methods 24 hours after reperfusion before thoracotomy, as 125 described in our previous study(24).

Measurement of PKA activity: PKA activity was measured utilizing non-radioactive PepTag assays (Promega, Southampton, Hampshire, UK) reliant upon a change in charge of the PepTag A1 peptide from +1 to -1 following phosphorylation (14). Sample reaction mixtures were incubated at room temperature for 15 minutes. After incubation, samples were separated on a $0.5 \%(\mathrm{w} / \mathrm{v})$ agarose gel at $100 \mathrm{~V}$ for $15-20$ minutes. Purified PKA catalytic subunit 131 served as a positive control, while the negative control contained only buffer. Bands were 132 visualized under ultraviolet light.

133 Quantification of Superoxide Production: Superoxide production in I/R heart tissue was measured by lucigenin-enhanced chemiluminescence as previously described(24). Superoxide production was expressed as relative light units (RLU) per second per mg heart 136 weight (RLU/s/mg wet tissue).

137 Determination of Total NOx Content in Cardiac Tissue: Cardiac tissue samples from AAR 
138 were rinsed, homogenized in deionized water (1:10, weight/volume), and centrifuged at $13914,000 \mathrm{~g}$ for 10 minutes. Tissue $\mathrm{NO}$ and its in vivo metabolic products $\left(\mathrm{NO}_{2}\right.$ and $\mathrm{NO}_{3}$, 140 collectively known as NOx) in the supernatant were determined via chemiluminescence NO 141 detector (SIEVER 280i NO Analyzer) as described in our previous study(24).

142 Quantitation of Tissue Nitrotyrosine Content: Ischemic/reperfused cardiac tissue nitrotyrosine 143 content (pmol of nitrotyrosine/mg protein), an accepted footprint of in vivo peroxynitrite 144 formation and a reliable index of nitrative stress, was determined via ELISA, described in our 145 previous publication $(22 ; 26)$.

Measurement of nuclear translocation of NF-kB p65: The relative increase of NF-kB p65 translocation into the nuclei of the ischemic/reperfused cardiomyoctyes was determined via ELISA per manufacturer's protocol (IMGENEX, San Diego, CA). In brief, cardiac tissue was cut into small pieces and homogenized. The mixture was centrifuged for 10 minutes at 10,000 rpm at $4{ }^{\circ} \mathrm{C}$. The supernatant represents the cytoplasmic fraction. The pellet was resuspended in nuclear lysis buffer, and subsequently centrifuged for 10 minutes at $14,000 \mathrm{rpm}$ at $4{ }^{\circ} \mathrm{C}$. This supernatant represents the nuclear fraction. A plate coated with anti-p65 antibody captured free p65 (both nuclear or cytoplasmic). Bound p65 was detected by adding a secondary antibody followed by alkaline phosphatase-conjugated secondary antibody. Each well's absorbance value was determined at $405 \mathrm{~nm}$ by a microplate reader (Molecular 156 Devices). The relative ratio of nuclear to cytoplasmic p65 was calculated by: (absorbance value of nucleus)/(absorbance value of cytoplasm) (16).

Western blot analysis: Protein from tissue homogenates was separated on SDS-PAGE gels, transferred to nitrocellulose membranes, and incubated with primary antibodies (against PKA

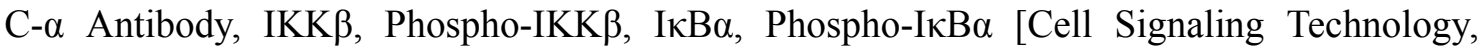
Danvers, MA], iNOS [Upstate, Chicago, IL], gp91phox [Transduction Laboratories, San Jose, $162 \mathrm{CA}])$ and horseradish peroxidase-conjugated secondary antibody. The blot was developed 
163 with a Supersignal Chemiluminescence detection kit (Pierce, Rockford, IL), and observed 164 with a Kodak Image Station 400 (Rochester, NY).

165 Statistical analysis: All values in the text and figures are presented as mean \pm standard error of 166 the mean of $\mathrm{n}$ independent experiments. All data (except Western blot density) were 167 subjected to one-way ANOVA followed by Bonferoni correction for post-hoc $t$ test. Western 168 blot densities were analyzed with the Kruskal-Wallis test followed by Dunn's post-hoc test. 169 Probabilities of 0.05 or less were considered to be statistically significant.

170 


\section{Results}

PKA deficiency blocked gAPN inhibition of MI/R-induced myocardial infarct, apoptosis, and cardiac dysfunction. MI/R did not alter PKA expression and slightly reduced PKA activity. Administration of APN 10 min before reperfusion had no effect upon PKA expression but significantly increased PKA activity (Figure 1) and reduced myocardial infarct size (Figure 2). To determine whether APN activation of PKA plays a causative role in APN cardioprotection, PKA expression was downregulated via intramyocardial siRNA injection. Pretreatment with the PKA-selective siRNAs reduced PKA expression by $70 \%(P<0.01$ relative to scramble group, n=6, Figure 2A). Cardiac specific PKA knockdown blocked APN-induced PKA activation (Figure 2B) and attenuated APN's infarct reduction effects (Figure 2C).

$\mathrm{MI} / \mathrm{R}$ resulted in significant cardiac injury including cardiac systolic and diastolic dysfunction (Figure 3) and myocardial apoptotic cell death (Figure 4). Consistent with our previous observations (22), gAPN administration significantly improved cardiac function after reperfusion (Figure 3, Scramble) and reduced apoptosis (Figure 4, Scramble). The knockdown of cardiac PKA expression (PKA siRNA) slightly worsened MI/R-induced cardiac dysfunction measured by LVEF and LVEDP (Figure 3A, B), but significantly increased infarct size (Figure 2C) and apoptosis (Figure 4A). More importantly, cardioprotective effect of APN was either completely abolished (anti-apoptotic effect on Figure 4) or significantly attenuated (Figure 2C, Figure 3) in the PKA-KD mice. Taken together, these results demonstrated that PKA activation plays a critical role in APN cardioprotection after MI/R.

PKA deficiency abolished the anti-oxidative and inhibited anti-nitrative effects of gAPN post $\underline{I / R}$. Having demonstrated that gAPN protects against reperfusion injury in a partially PKA-dependent manner, we investigate the underlying mechanisms potentially responsible. We report previously gAPN cardioprotection involves reduction of oxidative/nitrative stress 
196 (22). We performed two series of experiments assessing the relationship between PKA 197 signaling and oxidative/nitrative stress in $\mathrm{MI} / \mathrm{R}$ hearts. In the first experiment series, 198 MI/R-induced oxidative stress was detected. Under basal conditions, no significant difference existed between superoxide production in scramble and PKA-KD mice. MI/R induced greater increases in superoxide production in PKA-KD animals (Figure 5A). APN administration significantly inhibited MI/R-induced superoxide production in scramble animals (Figure 5A). However, APN treatment did not reduce superoxide production in PKA-KD animals (Figure 5A). We next identified potentially responsible molecular sources for the impaired 
PKA, iNOS-inhibitory effect of gAPN is not mediated by PKA.

Superoxide rapidly reacts with NO to form the cytotoxic molecule peroxynitrite. Nitrotyrosine (NT) content, the accepted footprint of peroxynitrite production, was increased by $\mathrm{MI} / \mathrm{R}$ injury both in scramble and PKA-KD mice (Figure 6C). Administration of gAPN significantly inhibited MI/R-induced NT generation in scramble animals, an effect that was partially blocked in PKA-KD animals (Figure 6C).

$g A P N$ decreased IKK/IKB/NF- $\kappa B$ signaling activation in $I / R$ myocardium, which was blocked by PKA deficiency. Decreased $\mathrm{I} \kappa \mathrm{B}$ phosphorylation preventing nuclear NF- $\kappa \mathrm{B}$ translocation attenuates myocardial reperfusion injury (17) and preserves cardiac function post MI. PKA

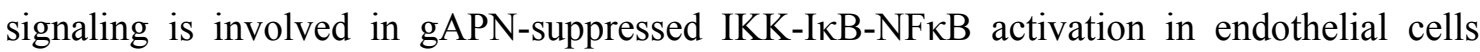
subjected to tumor necrosis factor- $\alpha$ or high glucose treatment (27). We investigated the possible mechanism underlying the PKA-dependent cardioprotective effects of gAPN, and

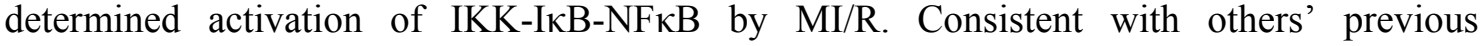
results(15; 30), MI/R induced significant IKK phosphorylation (Figure 7A), resulting in IкBa phosphorylation (Figure 7B) and NF- $\mathrm{BB}$ activation (Figure 7C) in both scramble and PKA-KD mice. MI/R-induced NFאB activation by $\mathrm{IKK} / \mathrm{I} \kappa \mathrm{B}$ phosphorylation was significantly inhibited by gAPN in scramble animals (Figures 7). However, gAPN did not inhibit NFאB activity during PKA-knockdown (Figure 7). These data suggest the

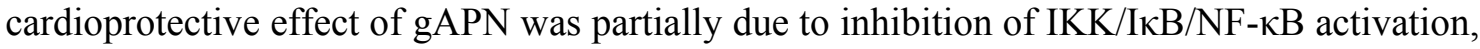
and such effect was dependent upon PKA signaling.

PKA-mediated cardioprotective effect of APN against MI/R injury is AMPK-independent:

Despite considerable evidence supporting the essential role of AMPK in adiponectin intracellular signaling $(4 ; 5 ; 7 ; 8 ; 11)$, we recently provided direct evidence of AMPK-independent gAPN-mediated cardioprotection in the intact animal $(24 ; 25)$. The current study suggests gAPN is cardioprotective in mice subjected to MI/R, with involvement 
246 of PKA signaling. We next investigated whether there is crosstalk between PKA and AMPK 247 pathways. Adult male mice overexpressing a dominant negative $\alpha 2$ subunit (D157A) of 248 AMPK (AMPK-DN) mice were pretreated with PKA siRNAs, and the effects of gAPN 249 administration upon MI/R injury were determined. Consistent with our previous data (24), $250 \mathrm{gAPN}$ inhibited MI/R-induced infarction (Figure 8A) and improved cardiac function (Figure 251 8B) in AMPK-DN animals. Importantly, PKA knockdown significantly attenuated this 252 protective effect, consistent with wild type (WT) mice results presented in Figures 1-3. 253 Moreover, PKA deficiency blocked the anti-oxidative/antinitrative effects of gAPN, 254 evidenced by augmented superoxide (Figure 8C) and peroxynitrite (Figure 8D) generation. 255 Together, these results support PKA-mediated gAPN cardioprotective effect against MI/R 256 injury is independent of AMPK signaling. 


\section{Discussion}

Several important observations have been made in the present study. Firstly, we demonstrate gAPN could not inhibit post-ischemic myocardial apoptosis, infarction, or cardiac dysfunction in cardiac specific PKA knockdown animals. This is the first evidence that PKA signaling mediates the cardioprotective effects of APN in ischemic heart disease in vivo. Secondly, we provide the first direct evidence that the anti-oxidative and antinitrative effects of gAPN in ischemic/reperfused hearts are mediated by PKA. Thirdly, we demonstrate PKA signaling is involved with adiponectin-mediated inhibition of

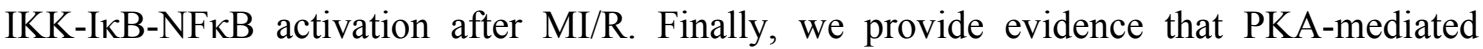
gAPN's cardioprotective effect against MI/R injury is independent of AMPK signaling.

APN is an abundant circulating adipocytokine secreted primarily from adipose tissue, exerting at least three major functions, including an insulin sensitization/metabolic-regulatory function (in the liver and muscle), an anti-inflammatory/vasculoprotective function, and an anti-ischemic/cardioprotective function. Numerous epidemiological studies underline the correlation between hypoadiponectinemic states and increased morbidity/mortality of cardiovascular ischemic diseases $(6 ; 12 ; 13 ; 18)$. Consistent with our and others' previous results, the present study confirms exogenous APN supplementation can significantly mitigate myocardial apoptosis, infarct size, and cardiac dysfunction.

The signal transduction pathway mediating the antioxidant effect of adiponectin remains intensely investigated. PKA is involved in adiponectin-mediated suppression of ROS and apoptosis in endothelial cells $(9 ; 19)$. Therefore, the current study sought to determine the role of PKA in gAPN's cardioprotective effect against MI/R injury, utilizing in vivo gene silencing techniques. In wild type mice, we demonstrate gAPN reversed MI/R-induced myocardial infarction, cardiomyocytes apoptosis, and cardiac dysfunction. However, in mice subjected to intramyocardial PKA knockdown, such protective effects of gAPN were either 
completely abolished or significantly attenuated. This is direct evidence that a significant portion of gAPN-mediated cardioprotection is PKA-dependent in intact animals.

Simultaneous overproduction of superoxide and NO not only causes inactivation of the cytoprotective NO, but generates the highly cytotoxic molecule peroxynitrite. Therefore, those therapeutic interventions preventing concomitant $\mathrm{NO}$ /superoxide overproduction effectively block peroxynitrite formation, and offer great tissue protection. The current study's results demonstrate that gAPN administration reduced synchronized NO/superoxide overproduction from NADPH oxidase and iNOS, and effectively block peroxynitrite formation in ischemic/reperfused hearts. Here, we demonstrate for the first time that gAPN inhibits I/R-induced oxidative in a PKA-dependent fashion, which may be the mechanism responsible for reduced cardioprotection by gAPN observed in PKA-deficient animals.

Nuclear factor kappa B (NF- $\mathrm{B})$ is a crucial transcription factor in the induction of genes involved in various physiological processes, including response to injury and inflammation. Activation of NF- $\kappa B$ requires phosphorylation of the inhibitor of NF- $\kappa B(I \kappa B)$ by IкB kinase (IKK), resulting $26 \mathrm{~S}$ proteasome-mediated degradation of $\mathrm{I} \kappa \mathrm{B}$. This allows the translocation of $\mathrm{NF} \kappa \mathrm{B}$ from the cytosol to the nucleus, where the heterodimer binds the promotor region of specific target genes. Activation of NF- $\kappa \mathrm{B}$ leads to the transcription of factors promoting inflammation (i.e. adhesion molecules, cytokines, and chemokines), but may also contribute to tissue remodeling, inflammation resolution, and the transcription of genes resulting in anti-inflammatory effects. Notably, NF-кB activation is implicated in the pathophysiology underlying acute myocardial infarction, heart failure, endothelial dysfunction, and unstable angina pectoris $(3 ; 10 ; 30)$. Furthermore, IKK inhibition attenuates myocardial injury and dysfunction following acute $\mathrm{I} / \mathrm{R}(30)$, and inhibition of I $\mathrm{KB}$ phosphorylation and resultant NF- $\mathrm{BB}$ activation attenuates $\mathrm{MI} / \mathrm{R}$ injury in cardiomyocytes. We currently demonstrate gAPN inhibits $\mathrm{IKK} / \mathrm{I} \kappa \mathrm{B} / \mathrm{NF} \kappa \mathrm{B}$ activation in the scramble heart. 
307 However, gAPN could not block MI/R-induced IKK and I $\mathrm{B}$ phosphorylation and resultant 308 NFKB activation in PKA-deficient mice. Together, these results indicate gAPN confers 309 cardioprotection via inhibition of $\mathrm{IKK} / \mathrm{I} \kappa \mathrm{B} / \mathrm{NF} \kappa \mathrm{B}$-mediated inflammation, and intact PKA 310 signaling is necessary for such gAPN-mediated anti-inflammatory protection against I/R 311 injury.

312 Substantial evidence supports the essential role AMPK plays in adiponectin 313 intracellular signaling. Pharmacological or genetic inhibition of AMPK activity virtually 314 abolishes the metabolic, anti-inflammatory and vasculoprotective effects of adiponectin. 315 However, we previously demonstrate APN-cardioprotection against MI/R injury is retained 316 in AMPK-DN mice. APN administration to AMPK-DN mice reduces MI/R-induced 317 oxidative and nitrative stress, indicating that AMPK-independent mechanisms mediate 318 gAPN's cardioprotective effect (24). Our present study data is consistent with our previous 319 findings. In AMPK-DN mice, the antioxidative/antinitrative effects (and consequent 320 cardioprotective effects) of gAPN were dependent upon whether PKA was silenced or not.

321 Our data supports the responsibility of PKA signaling in part for the protective actions of 322 adiponectin against MI/R injury.

323 In summary, we demonstrate for the first time that APN inhibits oxidative/nitrative 324 stress during MI/R via PKA signaling, in an AMPK independent fashion. This represents new 325 understanding in the intracellular signaling mechanisms of APN, as we continue to identify 326 novel therapeutic targets in the treatment of diabetic ischemic/reperfusion injury. 


\section{Acknowledgments}

329 This research was supported by the National Natural Science Foundation of China Grant 33081170144 (to XLW), 81170199 (to YJW) 81270185 (to JLZ), and the National Heart, Lung, 331 and Blood Institute Grants HL-63828 and HL-096686, the American Diabetes Association 332 Research Award 7-08-RA-98 (to XLM).

333

334

335 Author Disclosure Statement

336 No conflicts of interest, financial or otherwise, are declared by the authors.

337 


\section{Figure Legend}

Figure 1. The influence of APN upon PKA expression and activation. (A) Cardiac PKA expression in control (Sham MI/R) or MI/R treated with vehicle or gAPN. (B) Cardiac PKA activity in control or MI/R treated with vehicle or gAPN. $\mathrm{n}=6-9$ hearts/group. ${ }^{*} P<0.05$ vs. vehicle.

Figure 2. The influence of PKA signaling blockade by PKA-selective siRNAs upon myocardial PKA expression and activity and the resultant effect on myocardial infarct size. (A) Cardiac specific PKA knockdown decreased PKA expression. (B) PKA siRNAs significantly inhibited PKA expression. (C) PKA-specific siRNA significantly blocked gAPN inhibition of MI/R-induced myocardial infarction. $\mathrm{n}=9-12$ hearts/group. ${ }^{*} P<0.05,{ }^{*} * P<0.01$ vs. vehicle; ${ }^{\#} \mathrm{P}<0.05,{ }^{\# \#} P<0.01$ vs. scramble with the same treatment.

Figure 3. PKA deficiency blocked gAPN inhibition of MI/R-induced myocardial dysfunction. Cardiac function determined by echocardiography (A, LVEF) and left ventricular catheterization hemodynamic assay (B, LVEDP; C, +dp/dtmax). n=12-14 animals/group. ${ }^{*} P<0.05,{ }^{* *} P<0.01$ vs. vehicle; ${ }^{\#} \mathrm{P}<0.05,{ }^{\# \#} P<0.01$ vs. scramble with the same treatment.

\section{Figure 4. PKA deficiency blocked gAPN inhibition of MI/R-induced cardiomyocyte} apoptosis. (A) TUNEL staining; (B) caspase-3 activity. $\mathrm{n}=5-6$ hearts/group. ${ }^{* *} P<0.01$ vs. vehicle; ${ }^{\#} \mathrm{P}<0.05,{ }^{\#} P<0.01$ vs. scramble with the same treatment.

Figure 5. PKA deficiency blocked the anti-oxidative effects of gAPN post MI/R. (A) Production of superoxide ( $\mathrm{n}=6-7$ hearts/group); (B) gp91 $1^{\text {phox }}$ expression, representative 
363 Western blots shown ( $\mathrm{n}=5-6$ hearts/group). ${ }^{* *} P<0.01$ vs. vehicle; ${ }^{\#} \mathrm{P}<0.05,{ }^{\# \#} P<0.01$ vs. 364 scramble with the same treatment.

Figure 6. PKA deficiency partially blocked the anti-nitrative effects of gAPN post MI/R (A) NOx content ( $\mathrm{n}=5-6$ hearts/group); (B) Inducible nitric oxide synthase (iNOS) expression, representative Western blots shown ( $\mathrm{n}=5-6$ hearts/group); (C) Nitrotyrosine content, determined by ELISA ( $\mathrm{n}=6-8$ hearts/group). ${ }^{*} P<0.05, * * P<0.01$ vs. vehicle; ${ }^{*} \mathrm{P}<0.05$, ${ }^{\# \#} P<0.01$ vs. scramble with the same treatment.

Figure 7. gAPN decreased IKK/IкB/NF-кB signaling activation in $\mathrm{MI} / \mathrm{R}$ myocardium, which was blocked by PKA deficiency. (A) Phosphorylated IKK expression, representative Western blots shown ( $\mathrm{n}=-6$ hearts/group); (B) Phosphorylated I $\kappa$ B expression, representative Western blots shown ( $\mathrm{n}=5-6$ hearts/group); (C) Activated NFKB, determined by ELISA (n=6-8 hearts/group). ${ }^{*} P<0.05,{ }^{* *} P<0.01$ vs. vehicle; ${ }^{\#} \mathrm{P}<0.05,{ }^{\# \#} P<0.01$ vs. scramble with the same treatment.

Figure 8. PKA-mediated cardioprotective effect of APN against MI/R injury is AMPK-independent (A) Myocardial infarction, determined by Evans blue/TTC double staining ( $\mathrm{n}=9-12$ hearts/group); (B) Cardiac function, determined by echocardiography assay ( $\mathrm{n}=12-15$ animals/group); (C) Superoxide production ( $\mathrm{n}=6-8$ hearts/group); (D) Nitrotyrosine content, determined by ELISA ( $\mathrm{n}=6-8$ hearts/group). ${ }^{*} P<0.05,{ }^{* *} P<0.01$ vs. vehicle; ${ }^{\#} \mathrm{P}<0.05$, ${ }^{\# \#} P<0.01$ vs. scramble with the same treatment.

.


1. Bendall JK, Cave AC, Heymes C, Gall N and Shah AM. Pivotal role of a gp91phox-containing NADPH oxidase in angiotensin II-induced cardiac hypertrophy in mice. Circulation 105: 293-296, 2002.

2. Borchi E, Parri M, Papucci L, Becatti M, Nassi N, Nassi P and Nediani C. Role of NADPH oxidase in H9c2 cardiac muscle cells exposed to simulated ischaemia-reperfusion. J Cell Mol Med 13: 2724-2735, 2009.

3. Chandrasekar B, Smith JB and Freeman GL. Ischemia-reperfusion of rat myocardium activates nuclear factor-KappaB and induces neutrophil infiltration via lipopolysaccharide-induced CXC chemokine. Circulation 103: 2296-2302, 2001.

4. Chandrasekar B, Boylston WH, Venkatachalam K, Webster NJG, Prabhu SD and Valente AJ. Adiponectin Blocks Interleukin-18-mediated Endothelial Cell Death via APPL1-dependent AMP-activated Protein Kinase (AMPK) Activation and IKK/NF-\{kappa\}B/PTEN Suppression. J Biol Chem 283: 24889-24898, 2008.

5. Cheng KK, Lam KS, Wang Y, Huang Y, Carling D, Wu D, Wong C and Xu A. Adiponectin-induced endothelial nitric oxide synthase activation and nitric oxide production are mediated by APPL1 in endothelial cells. Diabetes 56: 1387-1394, 2007.

6. Combs TP, Pajvani UB, Berg AH, Lin Y, Jelicks LA, Laplante M, Nawrocki AR, Rajala MW, Parlow AF, Cheeseboro L, Ding YY, Russell RG, Lindemann D, Hartley A, Baker GR, Obici S, Deshaies Y, Ludgate M, Rossetti L and Scherer PE. A transgenic mouse with a deletion in the collagenous domain of adiponectin displays elevated circulating adiponectin and improved insulin sensitivity. Endocrinology 145: 367-383, 2004. 
7. Dyck JRB. The Ischemic Heart: Starving to Stimulate the Adiponectin-AMPK Signaling Axis.

Circulation 116: 2779-2781, 2007.

8. Fang X, Palanivel R, Cresser J, Schram K, Ganguly R, Thong FS, Tuinei J, Xu A, Abel ED and

Sweeney G. An APPL1-AMPK signaling axis mediates beneficial metabolic effects of adiponectin in the heart. Am J Physiol Endocrinol Metab 299: E721-E729, 2010.

9. Goldstein BJ, Scalia RG and Ma XL. Protective vascular and myocardial effects of adiponectin. Nat Clin Pract Cardiovasc Med 6: 27-35, 2009.

10. Grabellus F, Levkau B, Sokoll A, Welp H, Schmid C, Deng MC, Takeda A, Breithardt G and Baba HA. Reversible activation of nuclear factor-kappaB in human end-stage heart failure after left ventricular mechanical support. Cardiovasc Res 53: 124-130, 2002.

11. Hattori Y, Nakano Y, Hattori S, Tomizawa A, Inukai K and Kasai K. High molecular weight adiponectin activates AMPK and suppresses cytokine-induced NF-kappaB activation in vascular endothelial cells. FEBS Lett 582: 1719-1724, 2008.

12. Hui X, Lam KS, Vanhoutte PM and Xu A. Adiponectin and Cardiovascular Health: an Update. Br J Pharmacol 165: 574-590, 12 A.D.

13. Kawano T, Saito T, Yasu T, Saito T, Nakamura T, Namai K, Tamemoto H, Kawakami M, Saito M and Ishikawa SE. Close association of hypoadiponectinemia with arteriosclerosis obliterans and ischemic heart disease. Metabolism 54: 653-656, 2005.

14. Khaliulin I, Parker JE and Halestrap AP. Consecutive pharmacological activation of PKA and PKC mimics the potent cardioprotection of temperature preconditioning. Cardiovasc Res 88: 324-333, 2010.

15. Li C, Kao RL, Ha T, Kelley J, Browder IW and Williams DL. Early activation of IKK \{beta $\}$ during in 
16. Ohsugi T, Horie R, Kumasaka T, Ishida A, Ishida T, Yamaguchi K, Watanabe T, Umezawa K and

Urano T. In vivo antitumor activity of the NF-kappaB inhibitor dehydroxymethylepoxyquinomicin in a mouse model of adult T-cell leukemia. Carcinogenesis 26: 1382-1388, 2005.

17. Onai Y, Suzuki J, Kakuta T, Maejima Y, Haraguchi G, Fukasawa H, Muto S, Itai A and Isobe M. Inhibition of IkappaB phosphorylation in cardiomyocytes attenuates myocardial ischemia/reperfusion injury. Cardiovasc Res 63: 51-59, 2004.

18. Ouchi N, Shibata R and Walsh K. Cardioprotection by adiponectin. Trends Cardiovasc Med 16: 141-146, 2006

\section{Ouedraogo R, Wu X, Xu SQ, Fuchsel L, Motoshima H, Mahadev K, Hough K, Scalia R and}

Goldstein BJ. Adiponectin suppression of high-glucose-induced reactive oxygen species in vascular endothelial cells: evidence for involvement of a cAMP signaling pathway. Diabetes 55: 1840-1846, 2006.

\section{Shibata R, Sato K, Pimentel DR, Takemura Y, Kihara S, Ohashi K, Funahashi T, Ouchi N and} Walsh K. Adiponectin protects against myocardial ischemia-reperfusion injury through AMPK- and COX-2-dependent mechanisms. Nat Med 11: 1096-1103, 2005.

21. Simons M, Annex BH, Laham RJ, Kleiman N, Henry T, Dauerman H, Udelson JE, Gervino EV, Pike M, Whitehouse MJ, Moon T and Chronos NA. Pharmacological treatment of coronary artery disease with recombinant fibroblast growth factor-2: double-blind, randomized, controlled clinical trial. Circulation 105: 788-793, 2002. 
23. Tishinsky JM, Robinson LE and Dyck DJ. Insulin-sensitizing properties of adiponectin. Biochimie 94:

2131-2136, 2012.

24. Wang Y, Gao E, Tao L, Lau WB, Yuan Y, Goldstein BJ, Lopez BL, Christopher TA, Tian R, Koch

W and Ma XL. AMP-activated protein kinase deficiency enhances myocardial ischemia/reperfusion injury but has minimal effect on the antioxidant/antinitrative protection of adiponectin. Circulation 119:

$835-844,2009$.

25. Wang Y, Tao L, Yuan Y, Lau WB, Li R, Lopez BL, Christopher TA, Tian R and Ma XL.

Cardioprotective effect of adiponectin is partially mediated by its AMPK-independent antinitrative action. Am J Physiol Endocrinol Metab 297: E384-E391, 2009.

26. Wang Y, Wang X, Jasmin JF, Lau WB, Li R, Yuan Y, Yi W, Chuprun K, Lisanti MP, Koch WJ, Gao E and Ma XL. Essential role of caveolin-3 in adiponectin signalsome formation and adiponectin cardioprotection. Arterioscler Thromb Vasc Biol 32: 934-942, 2012.

27. Wu X, Mahadev K, Fuchsel L, Ouedraogo R, Xu SQ and Goldstein BJ. Adiponectin suppresses IkappaB kinase activation induced by tumor necrosis factor-alpha or high glucose in endothelial cells: role of cAMP and AMP kinase signaling. Am J Physiol Endocrinol Metab 293: E1836-E1844, 2007.

28. Xing Y, Musi N, Fujii N, Zou L, Luptak I, Hirshman MF, Goodyear LJ and Tian R. Glucose Metabolism and Energy Homeostasis in Mouse Hearts Overexpressing Dominant Negative \{alpha\}2 Subunit of AMP-activated Protein Kinase. J Biol Chem 278: 28372-28377, 2003.

\section{Yi W, Sun Y, Gao E, Wei X, Lau WB, Zheng Q, Wang Y, Yuan Y, Wang X, Tao L, Li R, Koch W and} Ma XL. Reduced Cardioprotective Action of Adiponectin in High-Fat Diet-Induced Type II Diabetic 
30. Zingarelli BF, - Hake PW FAU - Yang Z, Yang ZF, O'Connor MF, Denenberg AF and Wong HR. - 
A

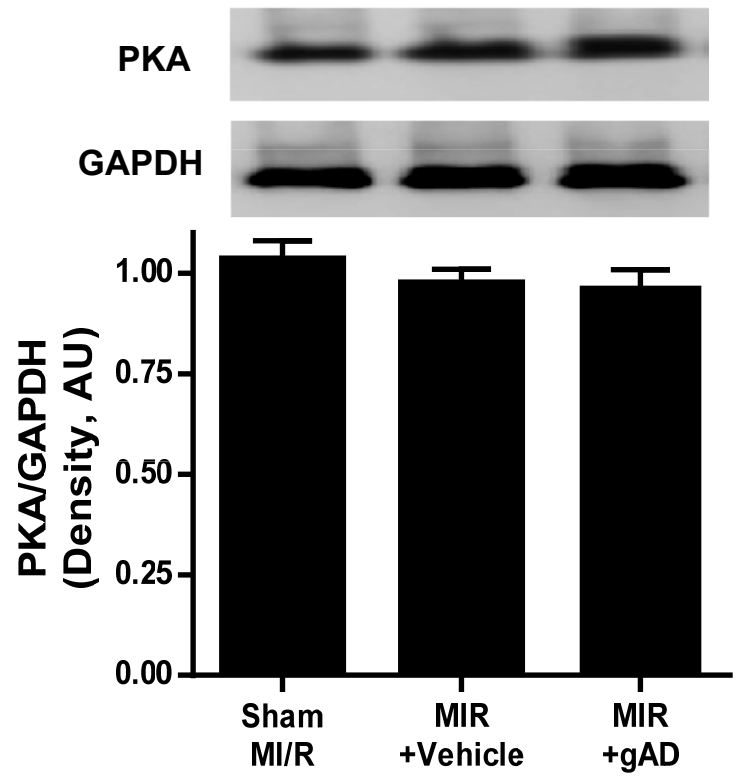

B
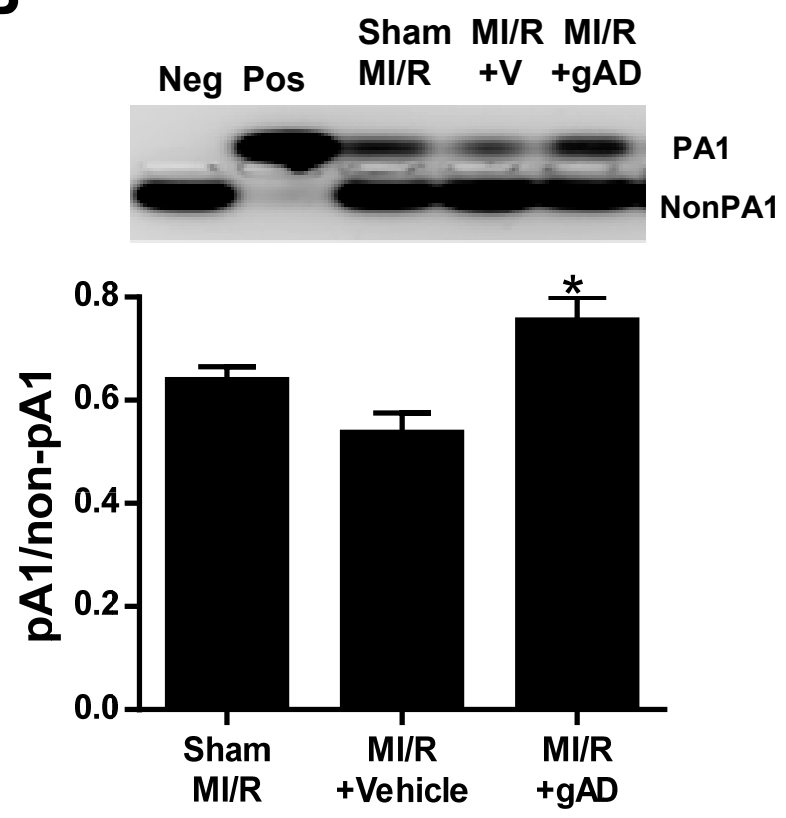
A

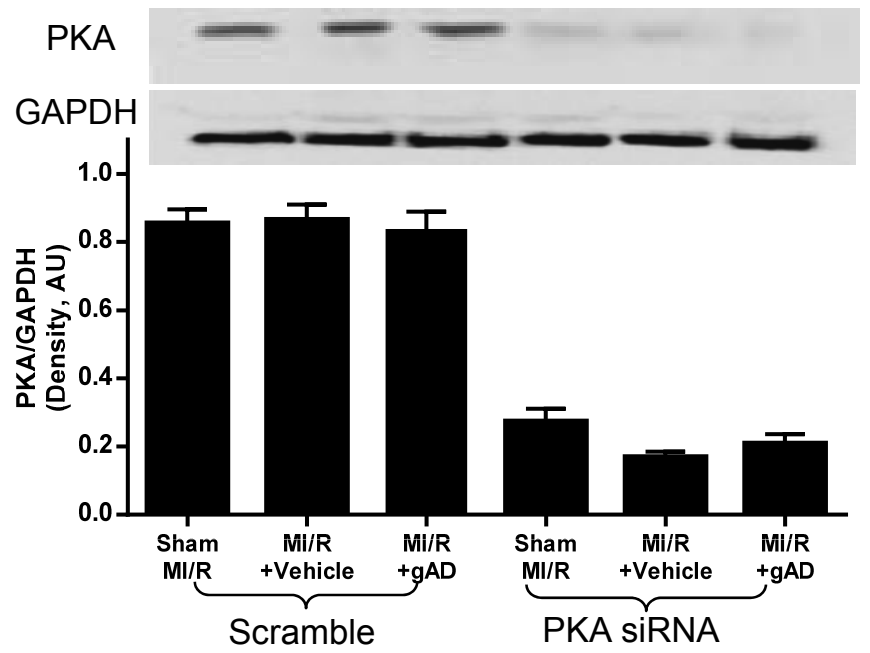

B

Phosph-A1

NonPhosph-A1
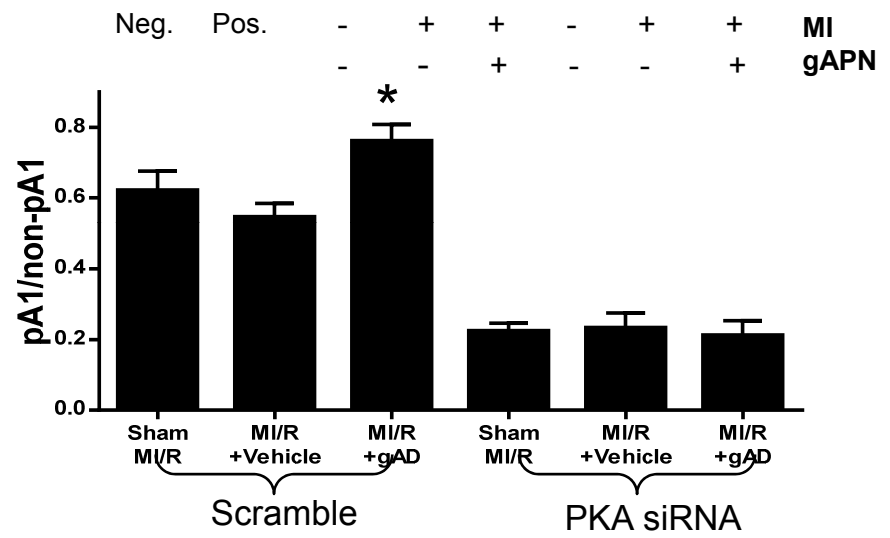

C

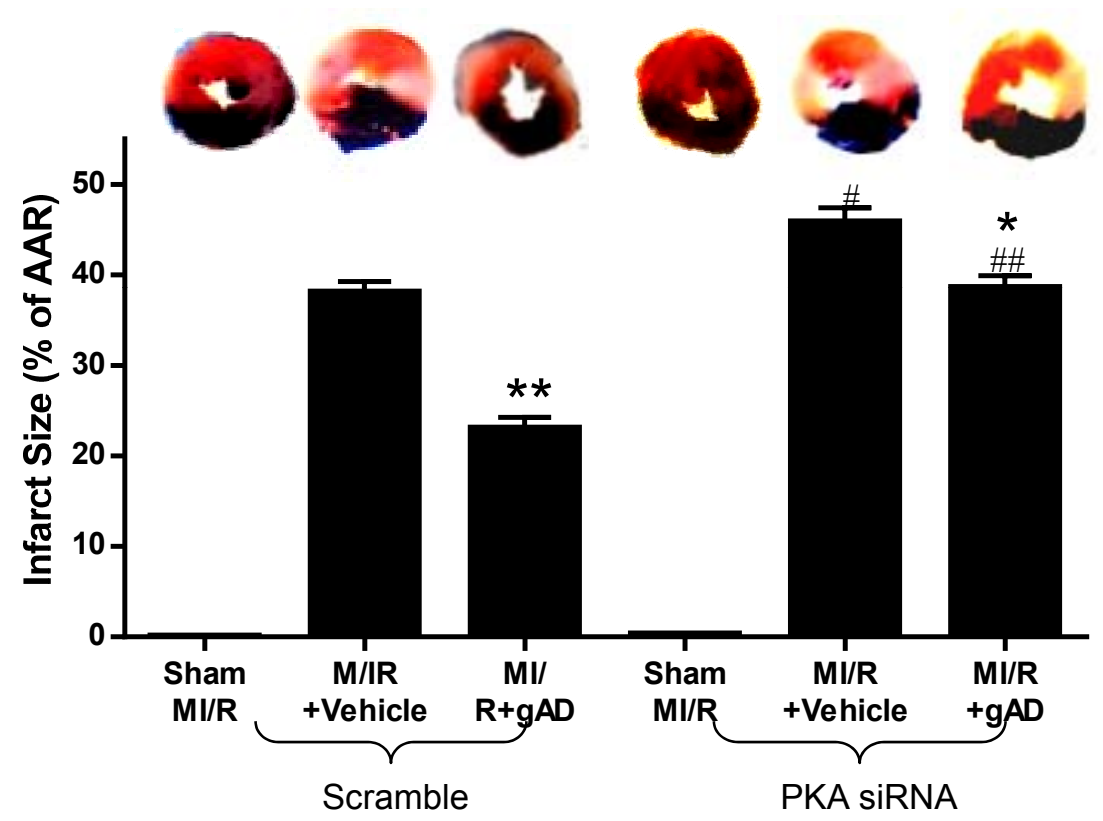

Fig 2 
A

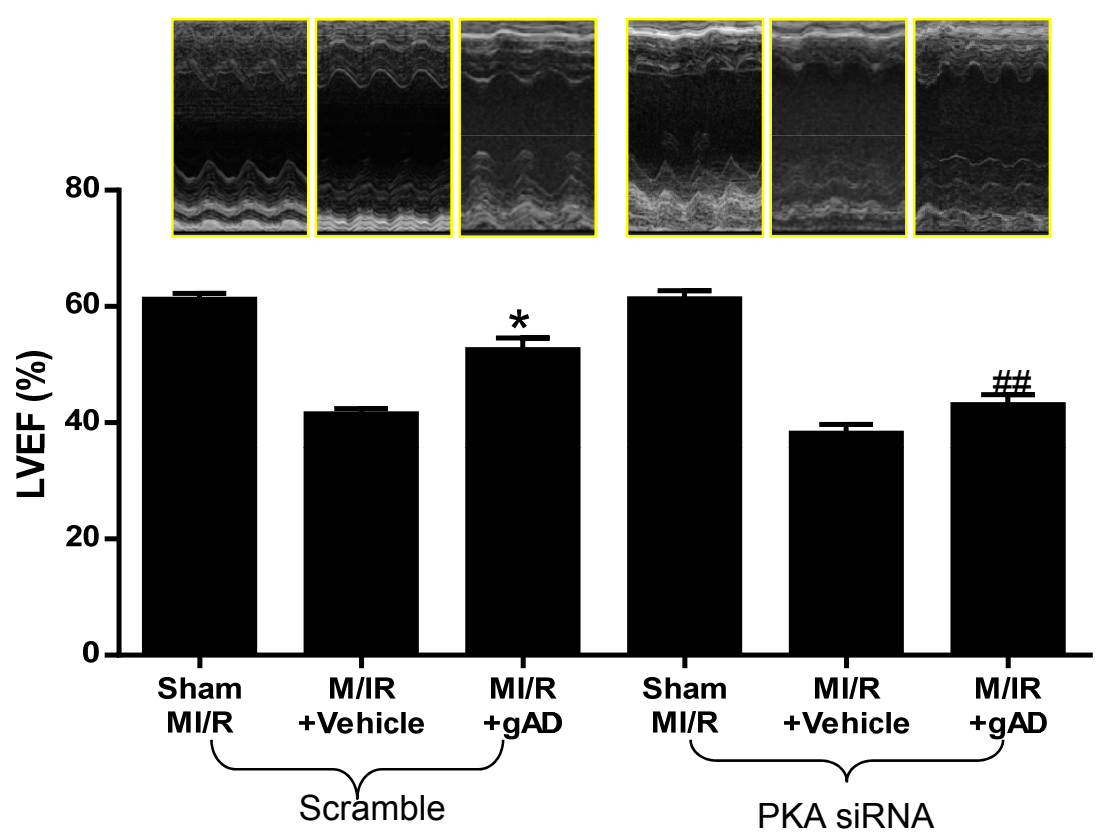

B

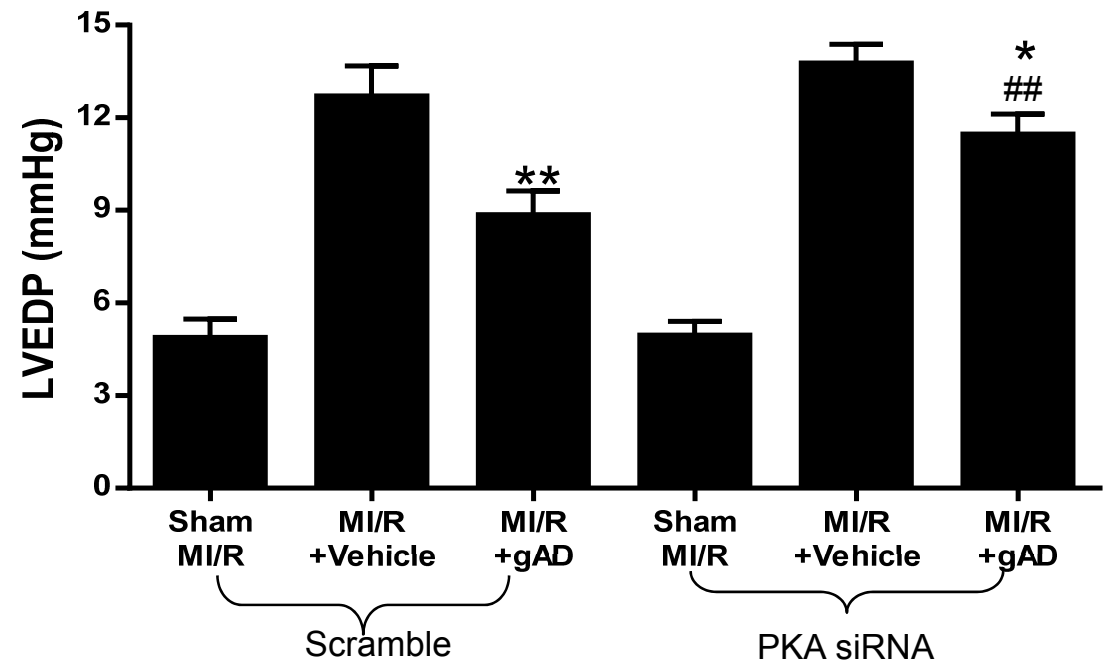

C

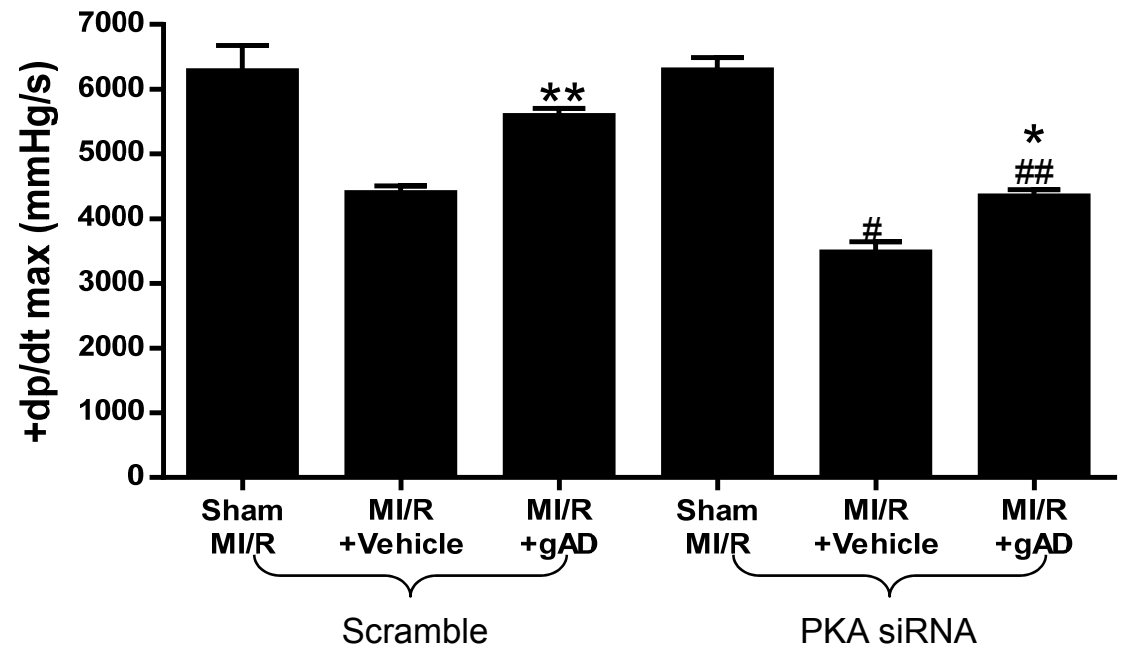

Fig 3 

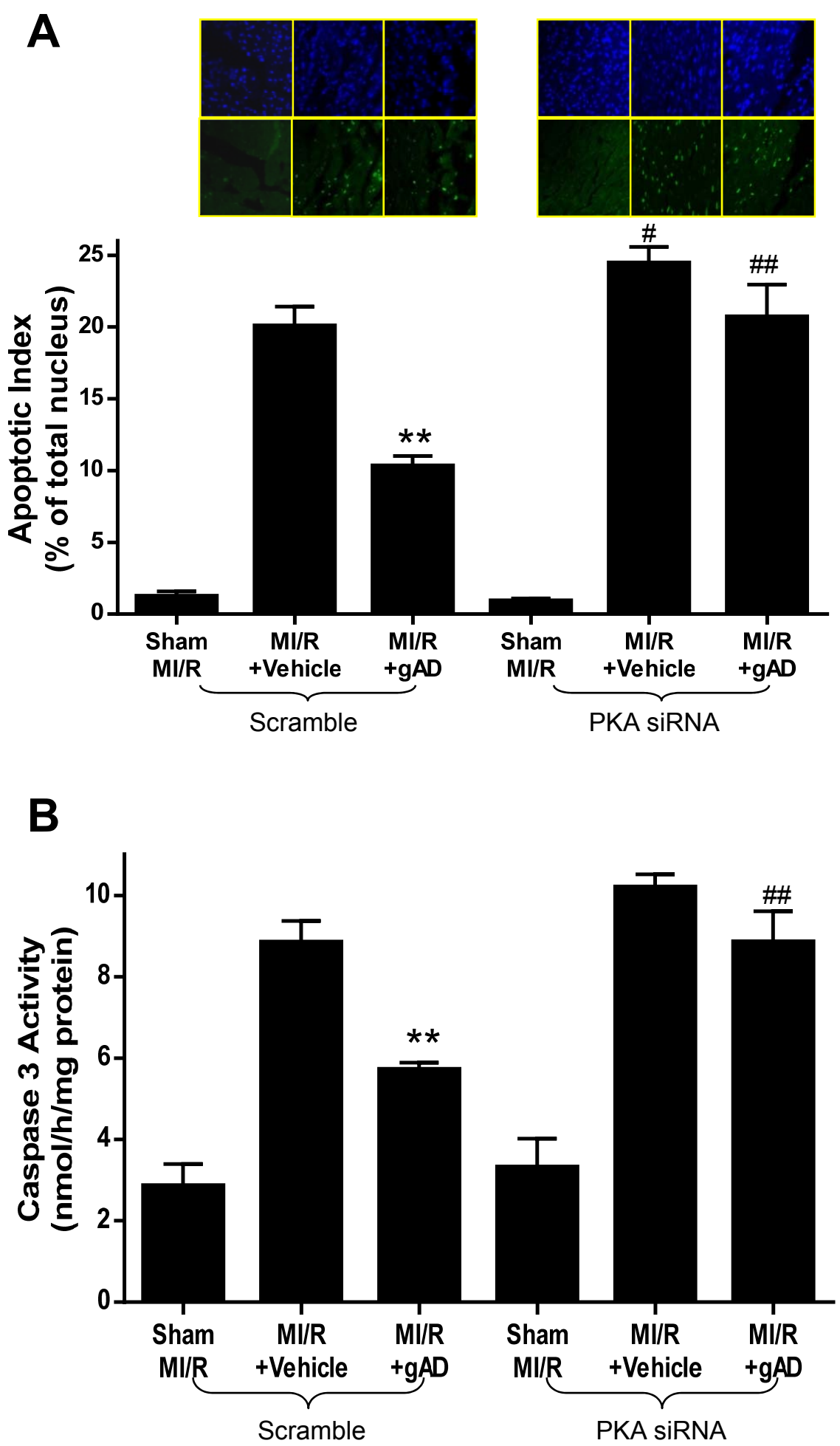

Fig 4 


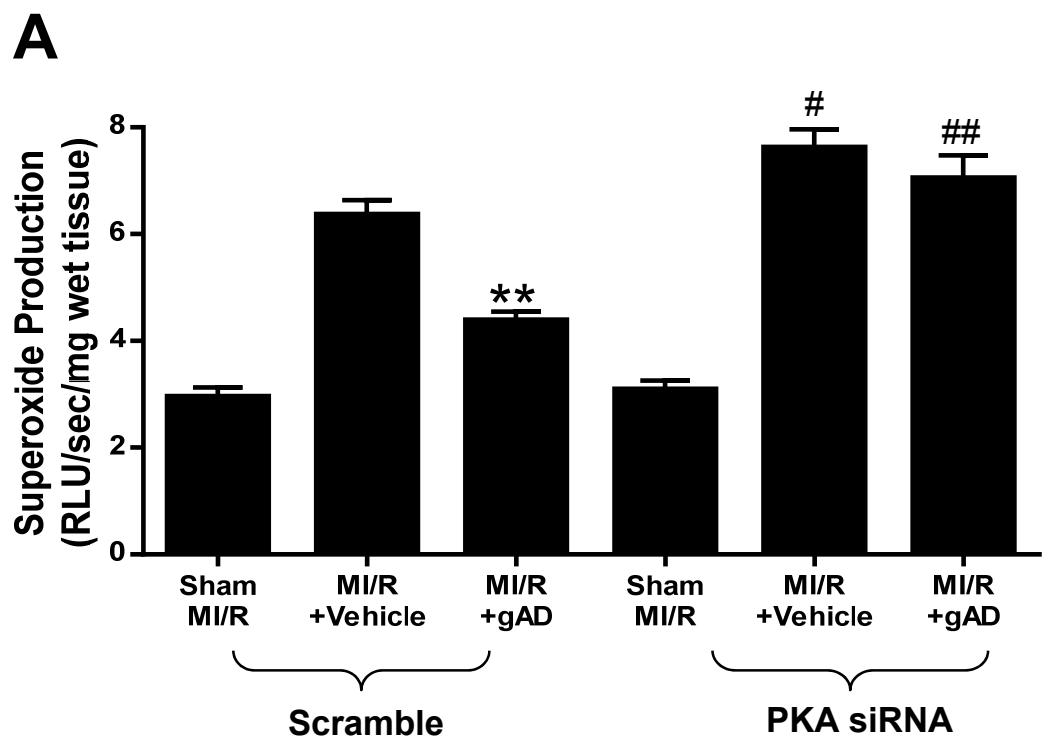

B

GAPDH

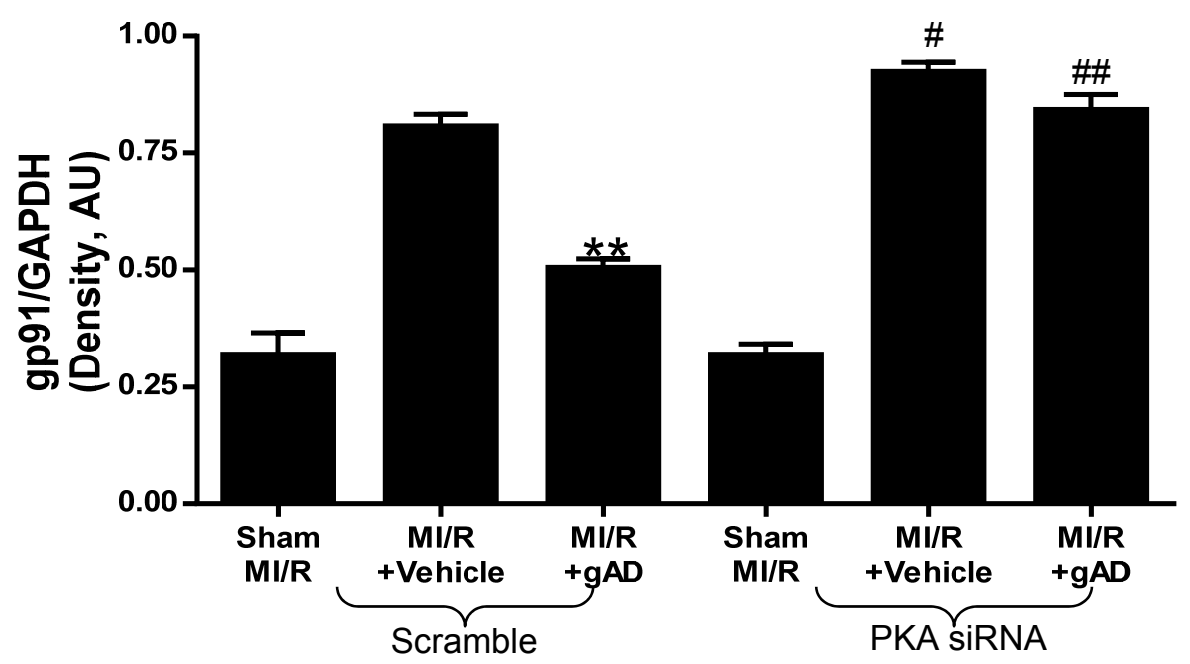

Fig 5 
A

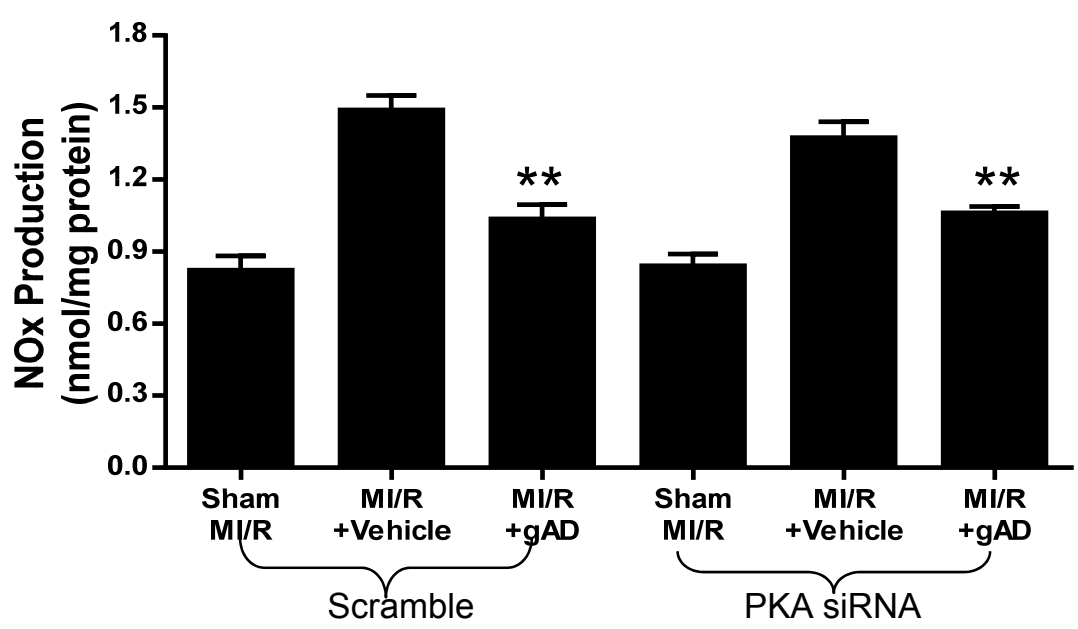

B

iNOS
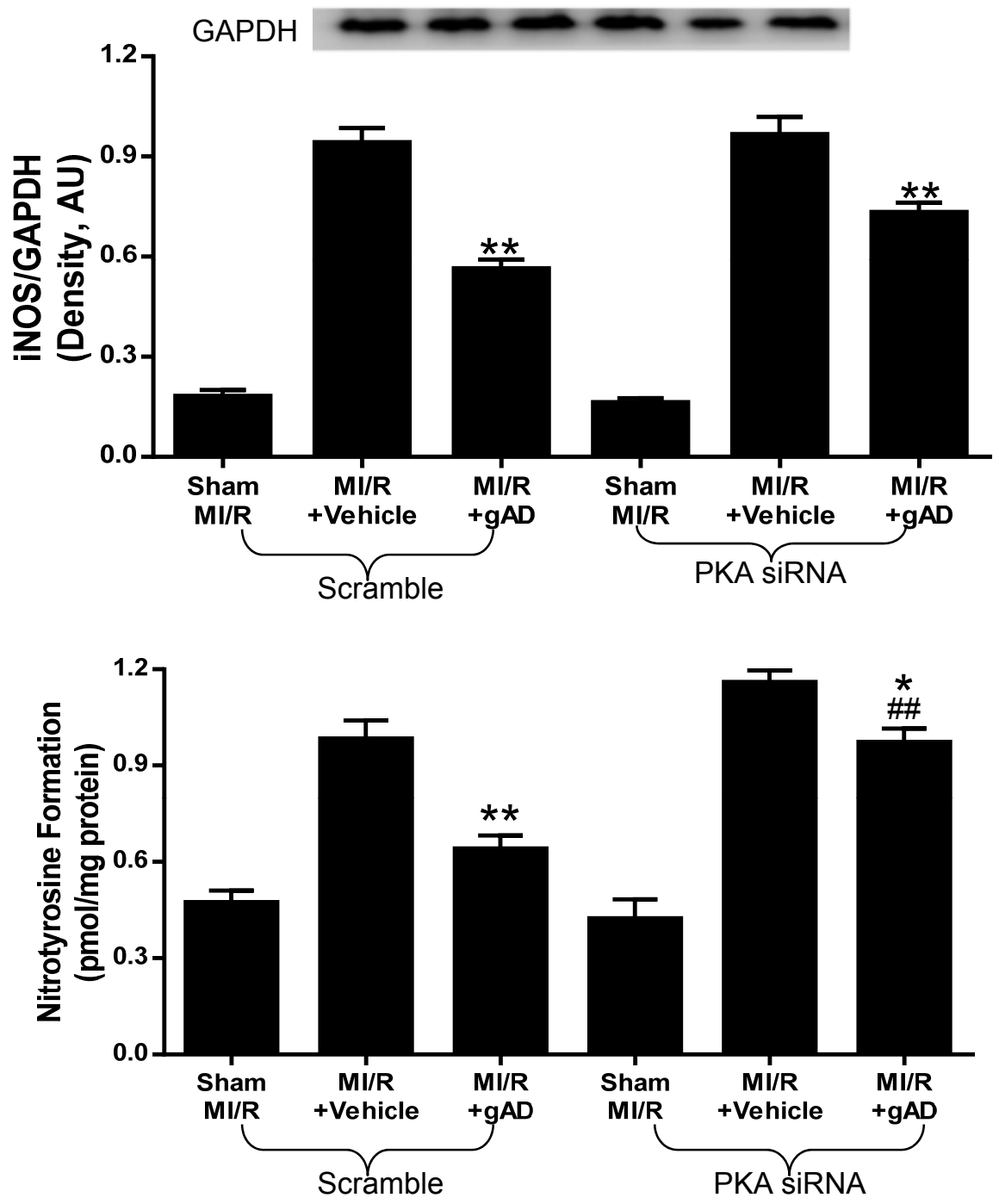

Fig 6 
A

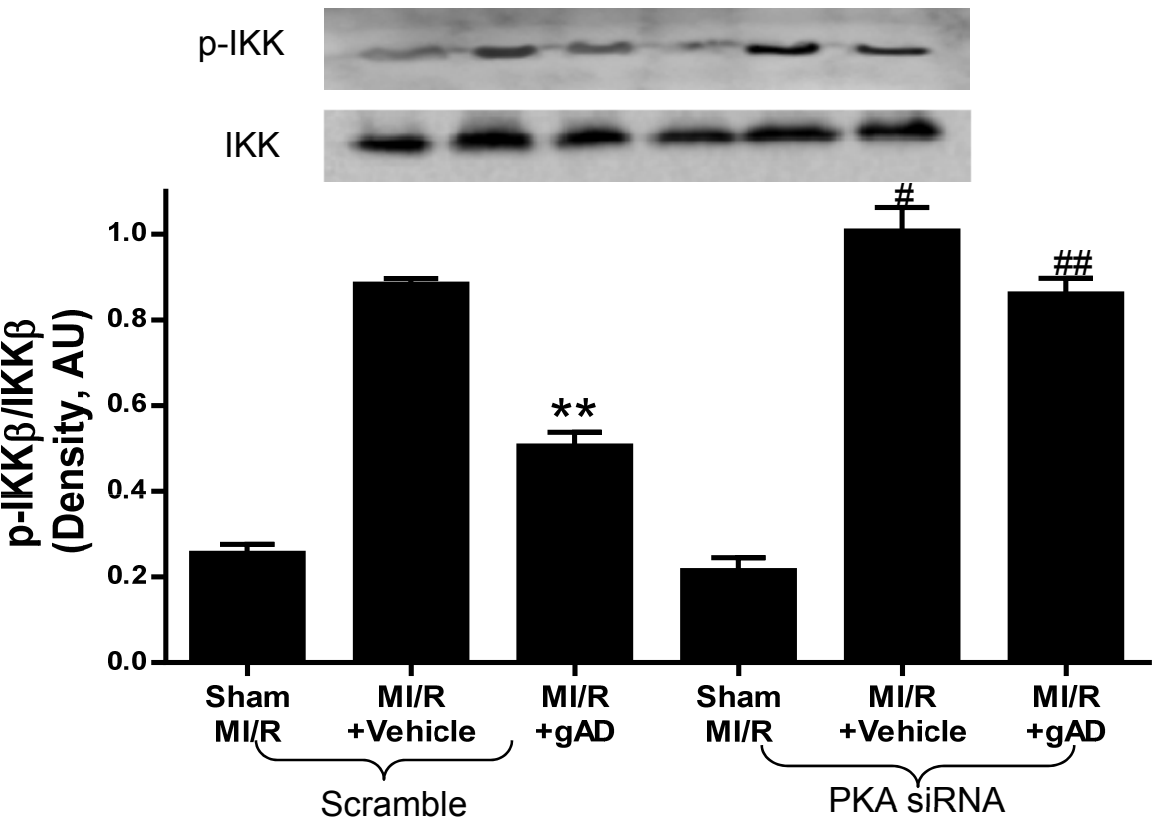

B

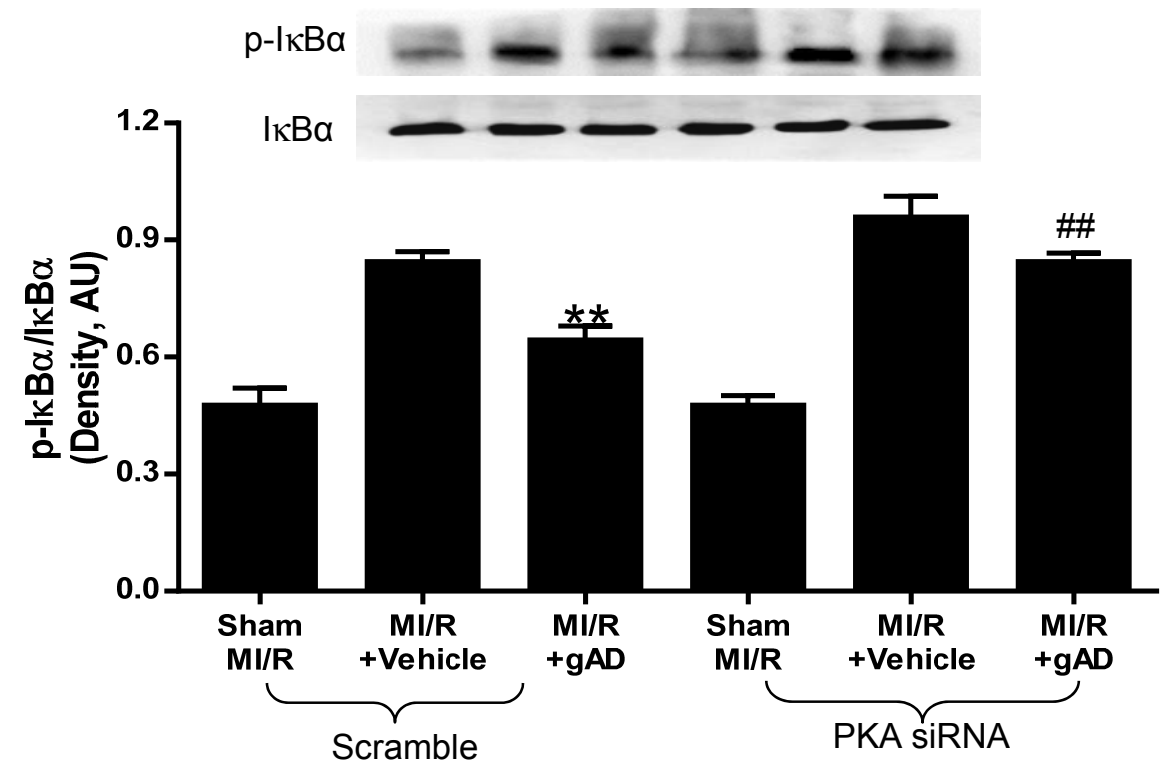

C

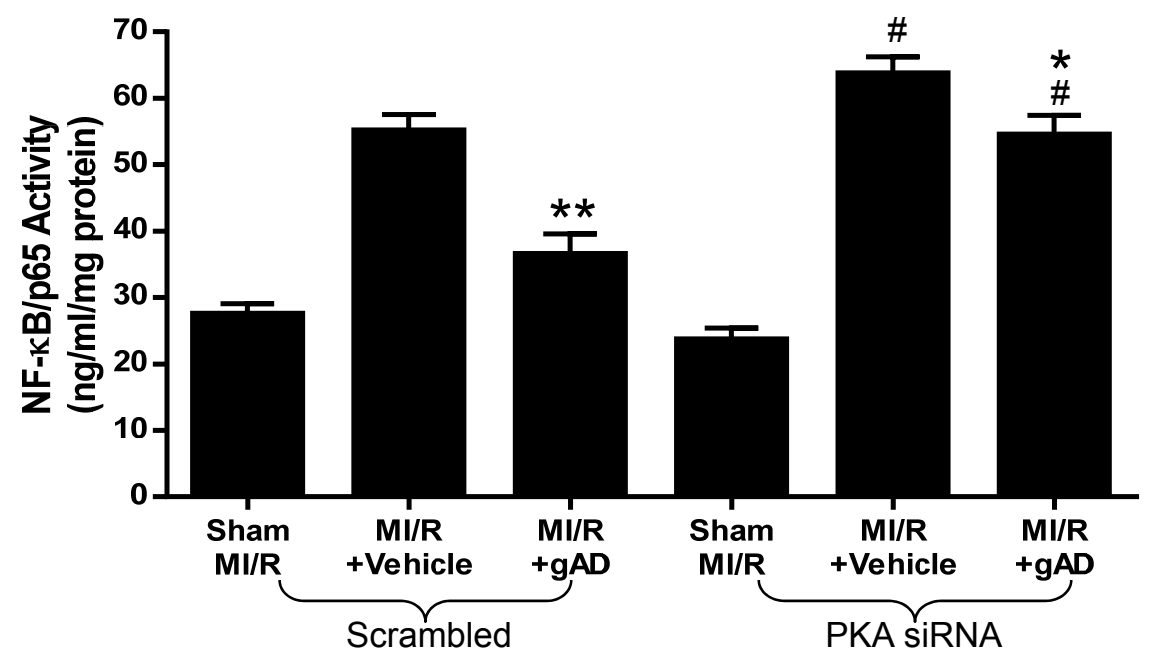

Fig 7 


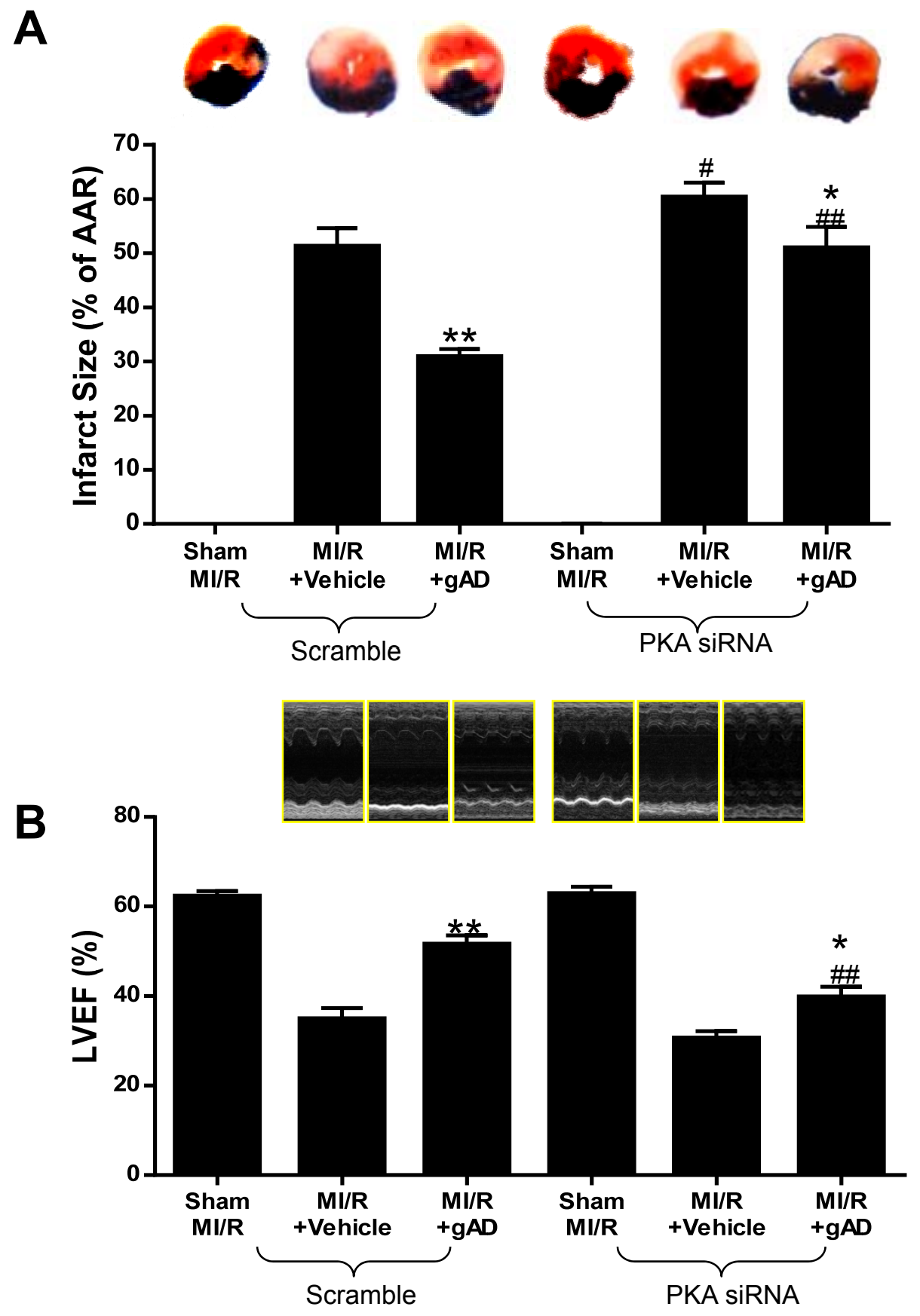

Fig 8 

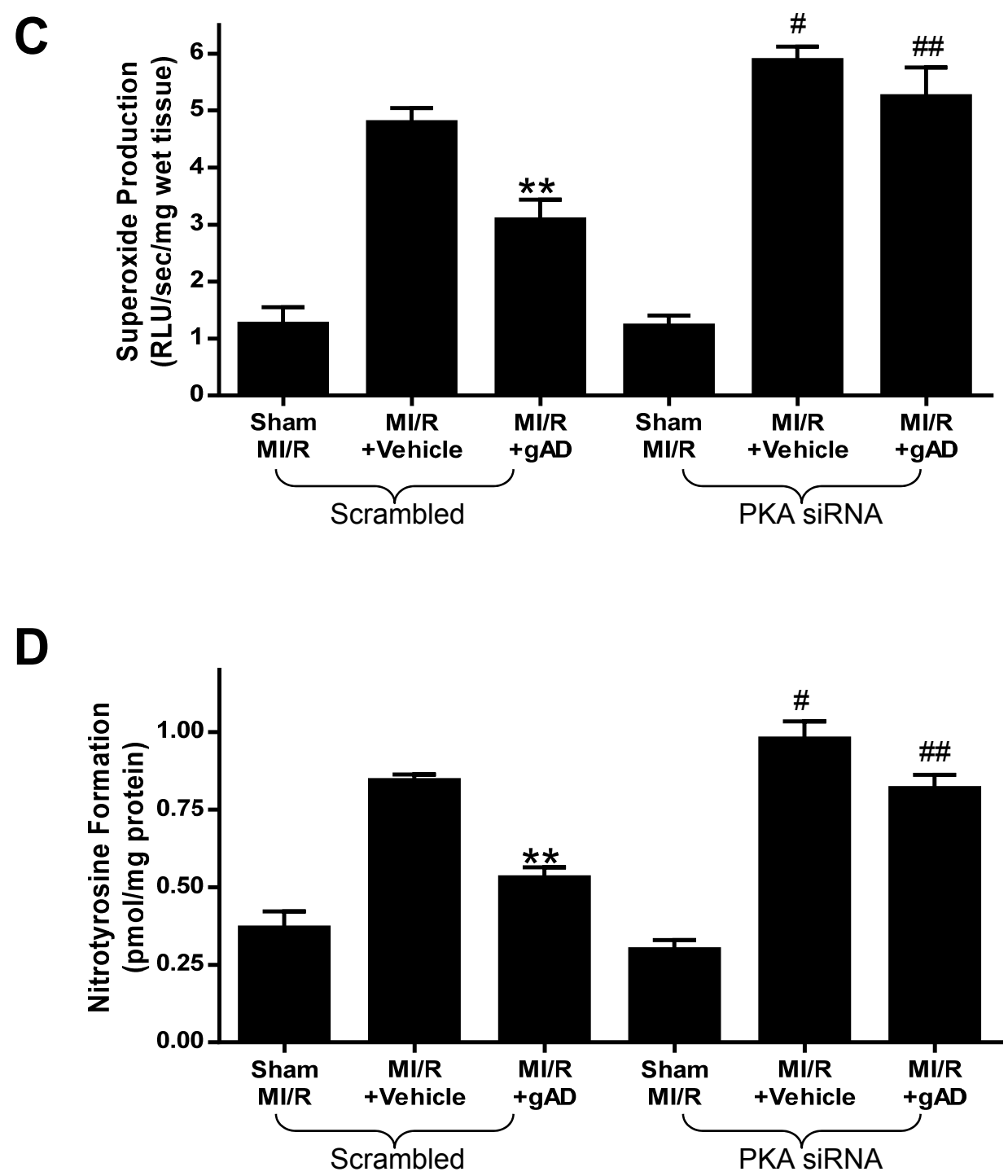

Fig 8 